\title{
Die alkalische Zersetzung der Hexachlorcyclohexane
}

\author{
Von Horst luther, Herbert Koelbel, Ernst Ruschenburg und Fritz Lampe \\ Aus dem Institut für Chem. Technologie der Technischen Hochschule Braunschweig und \\ dem Forschungslaboratorium der Chemischen Werke Rheinpreußen, Werk Homberg \\ (Z. Naturforschg. 4 b, 133-138 [1949]; eingegangen am 19. April 1949) \\ Als Grundlage für quantitative Analysen der isomeren Hexachlorcyclohexane mit \\ Hilfe der Raman-Spektren ihrer Gemische wurde der Gesamtgehalt an Hexachlorcyclo- \\ hexanen durch alkalische Zersetzung bei etwa $70-80^{\circ}$ bestimmt. Der Abspaltungs- \\ mechanismus an den einzelnen Isomeren, deren von Slade und Daasch angenommene \\ Struktur auch durch die Raman-Spektren wahrscheinlich gemacht werden konnte, wurde \\ diskutiert und trans-Abspaltung des Chlorwasserstoffs nach einer E2-Reaktion als be- \\ günstigt angesehen. Die sich dabei ergebende Reihenfolge der Zersetzungsgeschwindig- \\ keiten deckte sich mit eigenen Messungen und Literaturwerten. Die Endprodukte der \\ Zersetzung wurden ramanspektroskopiert.
}

$\mathrm{D}$ ie zunehmende Bedeutung deś $\gamma$-Hexachlorcyclohexans als Insektizid hat die Entwicklung einer brauchbaren qualitativen und quantitativen Analysenmethode für die bisher bestimmten isomeren Hexachlorcyclohexane stärker erforderlich gemacht.

Während van der Linden ${ }^{1}$ und $\mathrm{Slade}^{2}$ die einzelnen Isomeren im wesentlichen durch mühsame fraktionierte Kristallisation isolierten und analysierten, sind jetzt auch physikalisch-chemische Methoden zu ihrer Analyse ausgearbeitet worden.

Es sind u.a. zu nennen B ow en und P o g or els k in ${ }^{3}$ mit einer kryoskopischen Methode, A e p li und Mitarbeiter $4 \mathrm{a}$ sowie $\mathrm{Fuchs} 4 \mathrm{~b}$ mit der Verteilungschromatographie, Sch w a b e ${ }^{5}$ und D rag t ${ }^{6}$ mit der Polarographie, Kauer ${ }^{7}$ und $\mathrm{Daasch}{ }^{8}$ mit der Ultrarotspektroskopie, Goubea und $\mathrm{L} u \mathrm{th}$ e ${ }^{9}$ mit der Raman-Spektroskopie und schließlich eine Reihe von Bearbeitern des Problems mit einem Verfahren, das sich auf der verschiedenen Zersetzlichkeit der einzelnen Isomeren in Alkalien $\mathrm{zu}$ Trichlorbenzolen aufbaut. Unter ihnen seien $\mathrm{H}$ e r $\mathrm{r} m$ a $\mathrm{n} \mathrm{n}^{\mathbf{1 0}}$, Goldenson ${ }^{11}$, Gunther ${ }^{12}$, La Clair ${ }^{13}$ und besonders $\mathrm{Cristo \mathbf {1 } ^ { \mathbf { 1 4 } }}$ genannt. Der Vollständigkeit halber sei noch auf konventionelle Methoden hin-

1 T. v an der Linden, Ber. dtsch. chem. Ges. 45, 241 [1942].

2 R. E. Slade, Chem. and Ind. 40, 314 [1945].

3 C. V. B ow en u. M. A. Pogorelskin, Analytical Chem. 20, 346 [1948].

4a O. T. A e pli, P. A. M unter u. J. F. G a ll, Analytical Chem. 20, 610 [1948].

4b N. A. Fuchs, Chem. Zbl. 1948 II, 424 (Akad. Verl.).

5 K. Schw abe, Z. Naturforschg. 3 b, 5 [1948].

6 G. D r a gt, Analytical Chem. 20, 737 [1948].

7 K. C. K a u er, R. B. Du Vall u. F. N. A l q u i s t, Ind. Engng. Chem. 39, 1335 [1947].

8 L. W. Daasch, Analytical Chem. 19, 779 [1947]. gewiesen, die Rückschlüsse auf den $\gamma$-Gehalt aus der Wirksamkeit gegen Fliegen ${ }^{6}$, Kornkäfer, orientalische Küchenschaben oder Mikroorganismen ${ }^{15}$ ziehen.

Nachdem die Brauchbarkeit der ramananalytischen Methode durch Goubeau und Luther unabhängig voneinander festgestellt worden war, wurde dieses Verfahren auch zur Prüfung weiterer Fragen herangezogen ${ }^{9}$.

Die bisher ausgearbeiteten Methoden für die quantitative Auswertung der Raman-Analyse beruhen im wesentlichen auf der Bildung von Intensitätsverhältnissen bestimmter, für die zu analysierenden Substanzen charakteristischer Linien ${ }^{\mathbf{1 6}}$. Dieses Verfahren setzt also voraus, daß der Gehalt an einer Bezugssubstanz bekannt sein muß, um, von ihr ausgehend, den Gehalt an den übrigen Komponenten zu bestimmen.

In den technischen Hexachlorcyclohexangemischen können jedoch neben den Isomeren auch Produkte vorhanden sein, die einen anderen Chlo-

9 Über die quantitative Raman-Analyse der Hexachlorcyclohexane werden J. G o u be a u und H. Lu the r gemeinsam in einer gesonderten Arbeit berichten.

${ }_{10} \mathrm{R}$. H e r r m a n n, Chemische Werke RheinpreuBen, Homberg 1947, unveröffentlichte Versuche.

11 J. Goldenson u. S. Saß, Analytical Chem. 19, 320 [1947].

12 F. N. Gunther u. R. C. Blinn, J. Amer. chem. Soc. 69, 1215 [1947].

13 J. B. La Clair, Analytical Chem. 20, 241 [1948].

14 St. J. Cristol, J. Amer. chem. Soc. 69, 338 [1947].

15 R. Riemschneide r, Z. Naturforschg. 3b, 267 [1948].

16 J. Goubeau u. L. Thaler, Ver. Dtsch. Chem. Beiheft 41 [1941]; W. O t t i n g, Diss. Göttingen 1946; O. H. T h e i m e r, Diss. München 1944. 
rierungsgrad und eine andere Chlorierungsform besitzen, so daß zu bildende Intensitätsverhältnisse nicht auf einen Hexagehalt von $100 \%$, sondern auf einen geringeren Wert zu beziehen sind. Es war daher wichtig, eine Methode zu besitzen, die es gestattete, den Gesamtgehalt hexachlorierter Produkte zu bestimmen, ohne über die $\mathrm{Zu}$ sammensetzung im einzelnen etwas auszusagen.

\section{Die Gewinnung der Rein- komponenten}

Die für die Bestimmung der Raman-Spektren und die Durchführung der hier behandelten Versuche benötigten Rein-Isomeren wurden aus eigenen Chlorierungsprodukten isoliert. Unter den gewählten Versuchsbedingungen war ein Endprodukt angefallen, das etwa folgende Zusammensetzung besaß (bezogen auf eingesetztes Benzol):

15 Gew.-\% nicht umgesetztes Benzol,

60 Gew.- \% Kristallbrei (im wesentlichen $\alpha-, \beta-, \varepsilon-$ Komponente),

25 Gew.-\% Öl (im wesentlichen $\gamma$ - und $\delta$-Komponente).

Nach Abnutschen des Kristallbreies wurde das Benzol von dem Öl durch Destillation getrennt. Kristallbrei und öl wurden gesondert zur Entfernung von Benzolresten und kernchlorierten Produkten mitHeißdampf abgeblasen.

a) $\alpha$ - und $\beta$ - Komponente

Der Kristallbrei wurde mit Methanol gewaschen und bei Zimmertemperatur in einer Extraktionssäule mit Schwefelkohlenstoff extrahiert. Dabei ging das gesamte $\alpha$-Produkt mit geringen Teilen der $\beta$-Komponente in Lösung. Das zurückgebliebene $\beta$-Hexachlorcyclohexan wurde aus Benzol umkristallisiert. Schmp. $($ unter Sublimation $)=309^{\circ}$.

Der $\alpha$-reiche Extrakt wurde zur Abtrennung der letzten Reste $\beta$-Produkt nach Verdampfen des Lösungsmittels noch einmal in der Kälte extrahiert. Aus Alkohol-Eisessig ergab sich ein Endprodukt vom Schmp. $157,5^{\circ}$.

\section{b) $\varepsilon-\mathrm{Komponente}$}

Ka uer und Mitarbeiter ${ }^{7}$ berichteten zum erstenmal über die im Ultrarot verfolgte Anreicherung, die Gewinnung und die Eigenschaften des $\varepsilon$-Isomeren. Bej der ramanspektroskopischen Verfolgung der fortschreitenden Reinigung der $\alpha$ - von der $\beta$-Komponente war aufgefallen, daß neben der erwarteten Intensitätsschwächung der $\beta$-Linien ursprünglich der $\alpha$ Verbindung zugeschriebene Linien unsystematische Intensitätsschwankungen zeigten. Eine folgerichtige Untersuchung dieser Beobachtung ließ die Anwesenheit einer dritten Substanz vermuten. Diese konnte schließlich auch durch aufeinanderfolgende fraktionierte Umkristallisationen des anfallenden Kristall- breies aus Trichloräthylen, Chloroform, Äthylalkohol und Aceton rein dargestellt werden; sie schmolz bei $218^{\circ}$ und stand damit in guter Ubereinstimmung mit den Angaben von Kauer für das $\varepsilon$-Isomere.

\section{c) $\gamma$-Komponente}

Aus dem bei der Chlorierung entstandenen öl fiel beim Stehenlassen noch etwas $\alpha$-Hexachlorcyclohexan aus. Nach Abtrennung dieser Reste erstarrte das Öl nach etwa 8 Tagen weitgehend. Die nach Abnutschen gewonnenen Kristalle wurden nach van der Linden und Slade auf die $\gamma$-Komponente aufgearbeitet. Das Endprodukt wurde nacheinander noch mehrere Male aus Methanol und 80-proz. Eisessig umkristallisiert. Es zeigte sich dabei, daß zwar Schmelzpunktsänderungen nicht mehr auftraten, daß aber nicht derart intensiv gereinigtes Material im Raman-Spektrum noch Fremdlinien zeigte, die bisher noch nicht zugeordnet werden konnten, und daß in den Verunreinigungen auch die Träger einer störenden Fluoreszenz sowie des irrtümlich der $\gamma$-Verbindung zugeschriebenen Geruches waren. Das Endprodukt hatte einen Schmp. von $111,8^{\circ}$ und war absolut geruchlos.

d) $\delta$ - Ko mpone te

Das von den $\gamma$-Kristallen abgesaugte Öl wurde in sehr wenig Methanol aufgenommen; bei $5^{\circ}$ fielen nach längerem Aufbewahren kleine Nadeln aus, die aus Aceton und Tetrachlorkohlenstoff-Chloroform (1:1) umkristallisiert wurden. In noch stärkerem Maße als die $\gamma$-Komponente war das $\delta$-Hexachloreyclohexan von Fremdsubstanzen begleitet, welche die bei dem $\gamma$ Isomeren erwähnten Eigenschaften besaßen und darüber hinaus auch noch kristallisationsverzögernd wirkten. Oft war daher noch eine Intensivreinigung mit Aktivkohle und Chromatographie an Aluminiumoxyd notwendig, um ein spektroskopisch reines Produkt mit dem Schmp. $136^{\circ}$ zu gewinnen. Die unangenehmen Geruchstoffe des Gammexans reicherten sich in der $\delta$-Fraktion besonders stark an, waren jedoch auch dieser Komponente nicht eigen.*

\section{Die alkalische Zersetzung der $\mathrm{Hexachlorcyclohexane}$}

a) Grundlage n

Die alkalische. Zersetzung entsprechend der Umsetzung

$$
\mathrm{C}_{6} \mathrm{H}_{6} \mathrm{Cl}_{6}+3 \mathrm{NaOH}=\mathrm{C}_{6} \mathrm{H}_{3} \mathrm{Cl}_{3}+3 \mathrm{NaCl}+3 \mathrm{H}_{2} \mathrm{O}
$$

wurde von van der Linden ${ }^{1}$ an den $\alpha$-, $\beta$ und $\gamma$-Isomeren untersucht, um u. a. festzustellen, in welchem Verhältnis sich Trichlorbenzole bildeten. Als Hauptprodukt der Zersetzung erhielt van der Linden in allen Fällen das 1.2.4-Trichlor-

* Über Versuche zur Isolierung und Bestimmung der $\varepsilon$-Komponente ${ }^{\mathbf{1 7}}$ wird später ${ }^{\boldsymbol{9}}$ berichtet werden.

${ }^{17} \mathrm{~B}$ ast i a n sen u. H a s se l, Acta chim. Scand. 1, 683 [1947]. 
benzol. Daneben konnte er die beiden anderen isomeren Trichlorbenzole nachweisen. Je nach Wahl des die Salzsäure abspaltenden Mittels betrug der Anteil an 1.2.4-Trichlor-benzol 76-84\%.

Gunther und Blin $n^{12}$ fanden beim Kochen der reinen isomeren Hexachlorcyclohexane mit einem 80-proz. Überschuß an 1-n. KOH das 1.2.4Trichlor-benzol in einer Ausbeute bis zu 90\%.

Der qualitative und quantitative Nachweis der Trichlorbenzole ist mit $\mathrm{z}$. Tl. recht langwierigen Operationen verbunden. Es war daher naheliegend, spektroskopische Untersuchungsmethoden für die Bestimmung der Zersetzungsprodukte heranzuziehen. So führte K. C. Ka u e ${ }^{7}$ auch den Abbau des $\varepsilon$-Isomeren durch und fand im Ultrarotspektrum in der Hauptsache 1.2.4- neben kleinen Mengen 1.2.3-Trichlor-benzol. Die Entstehung von 1.3.5-Trichlor-benzol konnte nicht einwandfrei nachgewiesen werden.

Es wurden daher die sich bei der Zersetzung der Hexachlorcyclohexane bildenden Trichlorbenzole ramananalytisch untersucht. Die Spektren der reinen Trichlorbenzole sind bekannt. Ihre charakteristischen Linien unterscheiden sich soweit, daß die Erkennung und Bestimmung der drei Isomeren im Gemisch möglich ist.

Die Zersetzung, die im folgenden näher beschrieben ist, wurde mit $\alpha$ - und $\delta$-Hexachlorcyclohexan durchgeführt, um festzustellen, ob bei der Zersetzung des $\delta$-Hexachlorcyclohexans wesentliche Unterschiede im Vergleich mit den anderen Isomeren auftreten würden. Es wurden die auf dem beschriebenen Wege gewonnenen Reinsubstanzen eingesetzt.

\section{b) Versuchsbedingungen}

Für gewöhnlich wurde folgendermaßen zersetzt: In einem mit Rührer und Rückflußkühler versehenen, dreifach tubulierten Kolben wurden $10 \mathrm{~g} \alpha$ - bzw. $\delta$ Hexachlorcyclohexan in $200 \mathrm{~cm}^{3}$ absol. Methanol gelöst und unter ständigem Rühren bei konstanter Temperatur von $30^{\circ} 50 \mathrm{~g} \mathrm{KOH}$ im Laufe von $2 \mathrm{Stdn}$. zugefügt. Die durch Salzabscheidung trübe gewordene Flüssigkeit wurde danach zunäehst 6 Stdn. bei der genannten Temperatur gehalten und weitere 4 Stdn. bei der Siedetemperatur des Methanols unter anhaltendem Rühren gekocht. Anschließend wurde das Methanol bei $80 \mathrm{~mm} \mathrm{Hg}$ und bei einer Wasserbadtemperatur von +45 bis $50^{\circ}$ abdestilliert und zu dem zurückbleibenden Öl und Salz $400 \mathrm{~cm}^{3}$ Wasser hinzugefügt. Hierbei fielen die Trichlorbenzole aus. Sie wurden mit insgesamt $400 \mathrm{~cm}^{3}$ Äther extrahiert. Nach Trocknung der ätherischen Lösung wurde der Äther abgedampft und der Rückstand im Vakuum destilliert. Nach zwei- maliger Destillation über sehr wenig $\mathrm{P}_{2} \mathrm{O}_{5}$ ging eine wasserklare Flüssigkeit über: $\operatorname{Sdp}_{.21} 110,5^{\circ}$, Sdp.1 $80^{\circ}$. Von den erhaltenen Substanzen wurden Raman-Aufnahmen auf der Steinheilschen Raman-Apparatur hergestellt.

c) Die Zersetzungsprodukte des $\alpha$ und $\delta$-Hexachlorcyclohexans

Tab. 1 zeigt die Raman-Spektren der Zersetzungsprodukte des $\alpha$ - und $\delta$-Hexachlorcyclohexans und zugleich die Spektren der isomeren Trichlorbenzole nach K. W. F. K o h lra u s ch ${ }^{18}$. Die zusammengehörigen charakteristischen Bezugsfrequenzen sind gekennzeichnet.

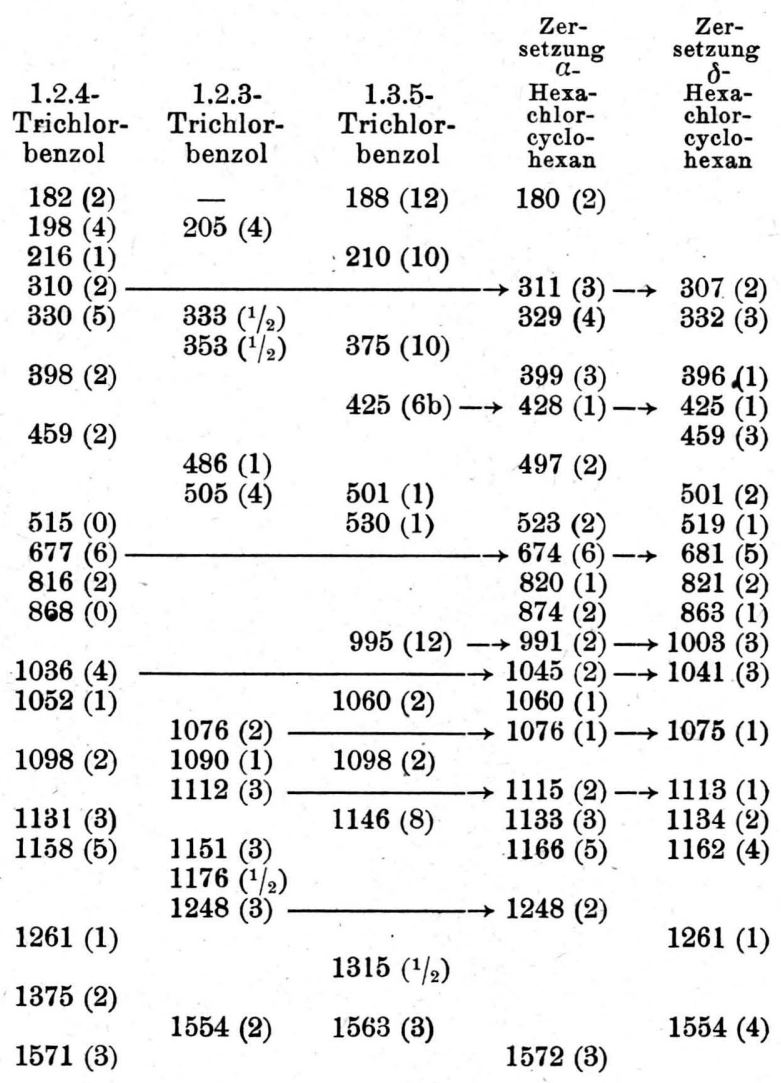

Tab. 1. Raman-Spektren der isomeren T'richlorbenzole und der Zersetzungsprodukte des $\alpha$ - und $\delta$-Hexachlorcyclohexans.

Der Tabelle ist $\mathrm{zu}$ entnehmen, daß sich in der Hauptsache 1.2.4-Trichlor-benzol neben geringen Mengen 1.2.3- und 1.3.5-Trichlor-benzol bildete.

Eine halb quantitative Abschätzung der $\mathrm{Zu}$ sammensetzung mit Hilfe der Intensitäten charak-

${ }^{18}$ K. W. F. Kohlrausch, Raman-Spektren, Leipzig 1943. 


\begin{tabular}{|c|c|c|c|c|c|}
\hline Beobachter & Subst. & $\begin{array}{c}1.2 .4- \\
\text { Trichlor- Trichlor- } \\
\text { benzol } \\
\text { in \% \% }\end{array}$ & $\begin{array}{c}1.3 .5- \\
\text { benzol } \\
\text { in \% }\end{array}$ & $\begin{array}{c}1.3 \text { - } \\
\text { benzor- } \\
\text { in \% }\end{array}$ & $\begin{array}{c}\text { Tem- } \\
\text { peratur } \\
\text { in }{ }^{\circ} \mathrm{C}\end{array}$ \\
\hline v. d. Linden & $a$ & 77,2 & 16,9 & 5,9 & 80 \\
eig. Messung & $\&$ & 80,0 & 15,0 & 10,0 & 30 \\
v.d. Linden & $\beta$ & 86,4 & 5,3 & 8,3 & 80 \\
v.d. Linden & $\gamma$ & 82,4 & 4,7 & 12,9 & 80 \\
eig. Messung & $\delta$ & 80,0 & 10 & $10-15$ & 30 \\
\hline
\end{tabular}

Tab. 2. Zersetzungsprodukte des $\alpha, \beta-, \gamma$ - und $\delta$-Hexachlorcyclohexans.

teristischer Linien ist in Tab. 2 den Werten von van der Linden gegenübergestellt.

Eine Zersetzung der $\delta$-Komponente wurde von van der Linden nicht durchgeführt.

Bei der Zersetzung der Hexachloreyclohexane ist der Anteil an gebildetem 1.2.4-Trichlor-benzol bei allen Komponenten annähernd gleich. Die Entstehung von 1.2.3-Trichlor-benzol gegenüber dem 1.3.5-Trichlor-benzol ist bei der $\alpha$-Komponente bevorzugt. Bei dem $\delta$-Isomeren ist es umgekehrt.

d) Die Kinetik der Z e rsetzung

Eingehend haben in neuerer Zeit I. B. La Cla i r, $\mathrm{K}$ a $\mathrm{u}$ e r und $\mathrm{Cr}$ is to $\mathrm{l}$ die Zersetzungsgeschwindigkeiten der verschiedenen Isomeren untersucht, die von der Temperatur und der Konzentration der Lösungen abhängen. In Tab. 3 sind ihre Ergebnisse mit den Resultaten eigener Versuche $\mathrm{zu}$ sammengestellt. Die Reihenfolge abnehmender Zersetzungsgeschwindigkeit geht also von der $\alpha$ - über die $\delta$-, $\gamma-, \varepsilon$ - zu der $\beta$-Komponente. Durch Temperatur- oder Konzentrationsänderungen wird die Absolut, aber nicht die Relativgeschwindigkeit der Zersetzung beeinflußt. Hierauf haben La Cla i ${ }^{13}$ sowie Herrman ${ }^{10}$ Analysenverfahren zur Bestimmung des Gehaltes einer Probe an $\beta-, \alpha+\delta$ - und $\gamma$-Hexachlorcyclohexan aufgebaut. Die Erklärung für die verschiedene Reaktionsgeschwindigkeit sieht Cristol darin, daß die Zersetzung unter Bevorzugung der trans-Abspaltung

\begin{tabular}{|c|c|c|c|c|c|c|c|c|c|}
\hline \multirow{2}{*}{ Beobachter } & \multicolumn{2}{|c|}{ Mengen (Mol) } & \multirow{2}{*}{ N } & \multirow{2}{*}{$\mid$\begin{tabular}{c|}
$\dot{\vec{\sigma}}$ \\
$\stackrel{0}{0}$ \\
$\stackrel{0}{ }$ \\
${ }^{2} \mathrm{C}$
\end{tabular}} & \multicolumn{5}{|c|}{$\begin{array}{l}\text { Prozentuale } \\
\text { Zersetzung }\end{array}$} \\
\hline & Hexachl. & $\mathrm{NaOH}$ & & & $a$ & $\beta$ & $\gamma$ & $\delta$ & $\varepsilon$ \\
\hline Christol & 0,001 & 0,005 & 60 & 20 & 100 & 0 & 45 & 80 & - \\
\hline Ka u e r & 0,007 & 0,04 & 300 & 0 & 100 & 0 & 52 & 75 & 42 \\
\hline La Clair & 0,00034 & 0,1667 & 10 & 0 & 90 & 0 & 43,7 & 83,3 & 32,8 \\
\hline eig. Messg. & 0,000 כั & 0,2667 & 10 & 20 & 100 & 0 & 45 & 82 & - \\
\hline
\end{tabular}

Tab. 3. Prozentuale Zersetzung der Hexachlorcyclohexane in Abhängigkeit von Zeit und Temperatur. der Salzsäure bei primärer Lockerung des Protons nach dem $\mathrm{E}_{2}$-Typus vor sich geht ${ }^{18,20}$. Nach $\mathrm{Hückel}$ würden dabei vornehmlich, sterische Momente die Abspaltung trans-ständiger Atome begünstigen, wie die beiden folgenden Schemen bei Aufhebung der freien Drehbarkeit um die C-C-Bindung (z. B. im Cyclohexan) zeigen:<smiles>[R]C([R])(Cl)C([R])([R])[Hg]O</smiles><smiles>[R]C([R])(Cl)C([R])([R])[Hg]O</smiles>

Bei cis-Stellung (I) würde das mit dem Proton auf der gleichen Seite liegende Chloratom das Anion abstoßen und daher die Abspaltung des Protons erschweren. Bei trans-Stellung (II) würde die Abstoßung geringer sein.

Cristol macht dazu noch geltend, daß diese Reaktionen zweiter Ordnung mit einer Waldenschen Umkehrung verbunden sind. Danach stellt sich die rasch verlaufende trans-Abspaltung folgendermaßen dar:

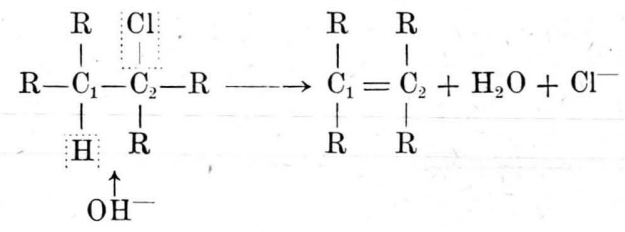

Das nach Lösung des Protons freiwerdende Elektronenpaar an $\mathrm{C}_{1}$ ruft bei gleichzeitiger $\mathrm{Ab}$ spaltung eines Chlorions die Umkehrung an $\mathrm{C}_{2}$ und die Bildung der Doppelbindung hervor.

Dagegen ist der Ablauf bei cis-Stellung ein anderer:
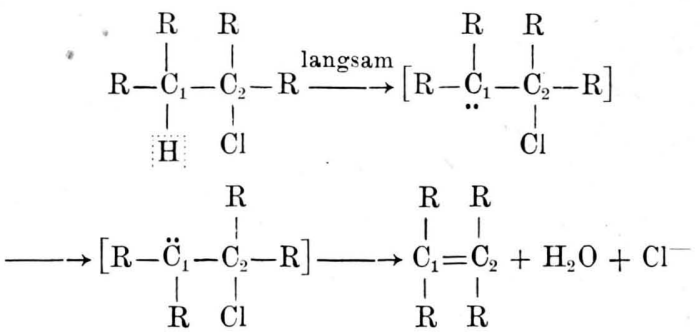

An $\mathrm{C}_{2}$ kann keine direkte Auslösung der Umkehrung erfolgen, sondern bei dem sich bildenden

19 E. D. $\mathrm{Hughes}$ u. Ch. K. In gold, J. chem. Soc. 1937, 1241; Trans. Faraday Soc. 37, 657 [1941].

${ }_{20}$ W. H i ckel, Naturforschung und Medizin in Deutschland 1939-1946, Bd.34, Wiesbaden 1948, S.116. 
Carbanion findet intermediär erst eine Umlagerung an $\mathrm{C}_{1}$ statt, ehe das Chlor an $\mathrm{C}_{2}$ abgespalten wird. Für nähere Einzelheiten beider Theorien muß auf die Originalliteratur verwiesen werden. Die Hauptreaktion zur Bildung des 1.2.4-Trichlorbenzols wird wahrscheinlich von beiden Einflüssen bestimmt werden.

Aus den erhaltenen Raman-Spektren der reinen Isomeren konnte zwar die Konfiguration der einzelnen Substanzen noch nicht einwandfrei bestimmt werden, da die Spektren, in Benzol und Tetrahydrofuran aufgenommen, besonders über $1000 \mathrm{~cm}^{-1}$ noch unvollständig sind. Es konnten aber andererseits keine Anhaltspunkte dafür gefunden werden, daß die Modellbilder von $\mathrm{Sl}$ a d e und $\mathrm{D}$ a a s $\mathrm{ch}$ falsch seien. Sie schienen vielmehr durch die Befunde gestützt zu werden. Der Verlauf der Abspaltung ist in Abb.1 für ein ebenes Cyclohexangerüst schematisiert wiedergegeben.

Bei Aufstellung der Abspaltungsmöglichkeiten ist berücksichtigt, daß bei Ablauf der nachfolgenden Reaktionen:

$$
\begin{aligned}
& \mathrm{C}_{6} \mathrm{H}_{6} \mathrm{Cl}_{6}+\mathrm{OH}^{-} \stackrel{k_{1}}{\longrightarrow} \mathrm{C}_{6} \mathrm{H}_{5} \mathrm{Cl}_{5}+\mathrm{Cl}^{-}+\mathrm{H}_{2} \mathrm{O} \\
& \mathrm{C}_{6} \mathrm{H}_{5} \mathrm{Cl}_{5}+\mathrm{OH}^{-} \stackrel{k_{2}}{\longrightarrow} \mathrm{C}_{6} \mathrm{H}_{4} \mathrm{Cl}_{4}+\mathrm{Cl}^{-}+\mathrm{H}_{2} \mathrm{O} \\
& \mathrm{C}_{6} \mathrm{H}_{4} \mathrm{Cl}_{4}+\mathrm{OH}^{-} \stackrel{k_{3}}{\longrightarrow} \longrightarrow \mathrm{C}_{6} \mathrm{H}_{5} \mathrm{Cl}_{3}+\mathrm{Cl}^{-}+\mathrm{H}_{2} \mathrm{O}
\end{aligned}
$$

- von denen für das $\alpha$ - und $\gamma$-Isomere nach Cristol $k_{1}$ für die gesamte Dehydrochlorierung geschwindigkeitsbestimmend ist - das zweite Proton in $o-, p$ - oder $m$-Stellung zum ersten gelockert, und daß auch der Angriff auf das dritte entsprechend festgelegt werden kann. $o$ - und $p$ Abspaltungsreihenfolge liefern in gleicher Weise 1.2.4-Trichlor-benzol. Sie werden bei der Protonenlockerung bevorzugt sein. $m$-Verlauf wird vorwiegend 1.3.5- neben geringen Mengen 1.2.4-Trichlor-benzol liefern. Die Bildung von 1.2.3-Trichlor-benzol ist in jedem Fall nur durch einen grundsätzlich anderen Reaktionsmechanismus oder durch nachträgliche Umlagerung denkbar. Versuche, letztere im Modellversuch unter den üblichen Zersetzungsbedingungen nachzuweisen, blieben erfolglos.

Die Zersetzungsgeschwindigkeit läuft der Zahl der vorhandenen trans-Abspaltungsmöglichkeiten in den Isomeren völlig parallel. Die $\alpha$ - und $\delta$-Komponenten besitzen bei der Zersetzungsgeschwindigkeit 100 bzw. 80 zwei, die $\gamma$ - und $\varepsilon$-Komponenten bei der Zersetzungsgeschwindigkeit $40-45$
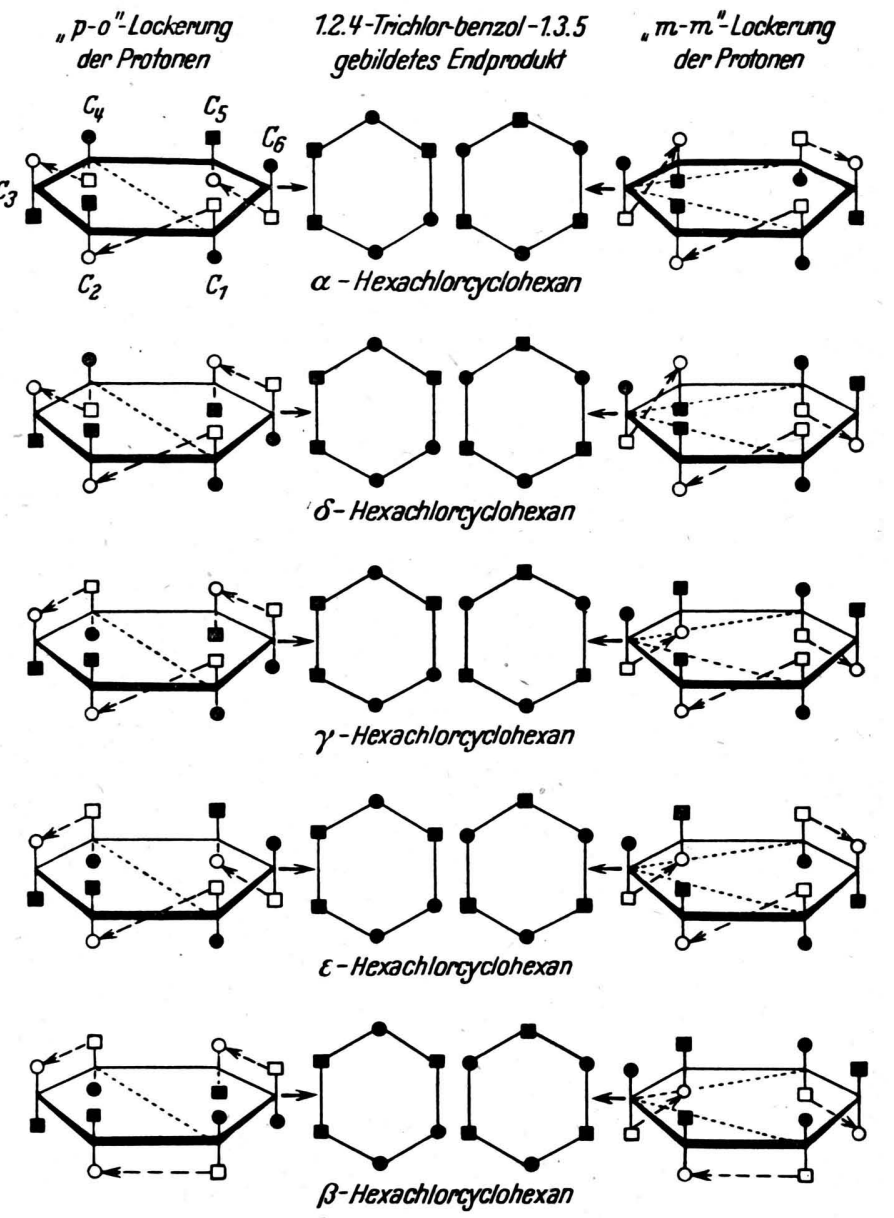

Abb. 1. Abspaltungsschema der isomeren Hexachlorcyclohexane.

$$
\begin{gathered}
\left.\begin{array}{l}
\square=\mathrm{H} \\
\mathrm{O}=\mathrm{Cl}
\end{array}\right\} \text { abgespalten; } \quad \mathrm{H} \\
\begin{array}{c}
\mathrm{H} \\
--\rightarrow \text { Abspaltungsrichtung. }
\end{array}
\end{gathered}
$$

eine und die $\beta$-Komponente bei der Zersetzungsgeschwindigkeit 0 keine trans-Abspaltungsmöglichkeit.

Bis zu einem gewissen Grade beweisen sich also trans-Abspaltungstheorie und angenommene Strukturen gegenseitig. Der gleichmäßig hohe Gehalt an 1.2.4-Trichlor-benzol unter den Zersetzungsprodukten aller Isomeren macht es unwahrscheinlich, daß evtl. in dem einen oder anderen Isomeren mehrere Chloratome an einem Kohlenstoffatom sitzen. $\mathrm{Zu}$ dem gleichen Resultat führt die beobachtete Konstanz der C-Cl-Schwingung in den Spektren der Hexachlorcyclohexane. Die feinen Unterschiede in dem Anteil des 1.3.5und 1.2.3-Trichlor-benzols an den Zersetzungs- 
produkten lassen sich qualitativ auch noch aus dem angenommenen Abspaltungsmechanismus ableiten. Bevor jedoch nicht mehrfach bestätigte Analysenwerte vorliegen, sind Schlüsse in dieser Richtung unsicher.

III. Bestimmung des Gesam tgehaltes an Hexachlorcyclohexanen durch die alkalische Zersetzung

Nach den Ergebnissen der vorangegangenen Untersuchungen wurde schließlich folgende Vorschrift für die Bestimmung des Gesamtgehaltes an Hexachlorcyclohexanen ausgearbeitet:

$2,0 \mathrm{~g}$ Substanz werden in einen $250-\mathrm{cm}^{3}$-Schliffkolben mit $20 \mathrm{~cm}^{3}$ Methanol gespült und $20 \mathrm{~cm}^{3}$ einer $2-n$. methanolischen Kalilauge tröpfenweise zugegeben. Anschließend wird 2 Stdn. bei leichtem Sieden unter Rückfluß gekocht. Nach dem Erkalten wird mit Wasser nachgespült und mit 2-n. Schwefelsäure auf Phenolphthalein titriert.

Entsprechend den Feststellungen von Goldenson und $\mathrm{Sa} B$ ergaben sich bei Vorliegen von technischen Chlorierungsprodukten, die mit Wasserdampf behandelt waren, nach Volhard keine starken Abweichungen.

Die Ergebnisse der quantitativen Raman-Analysen, die meist nur den Gehalt an $\alpha$ - und $\gamma$-Komponente bestimmten, wurden dann auf den Gesamtgehalt an Hexachlorcyclohexanen bezogen.

\title{
Über die Abgabe von Kernstoffen in das Protoplasma der menschlichen Leberzelle*
}

\author{
Von Hans-Werner Altmann \\ Aus dem Pathologischen Institut der Universität Freiburg i. Br. \\ (Z. Naturforschg. 4 b, 138-144 [1949]; eingegangen am 19. Januar 1949)
}

\begin{abstract}
Untersuchungen an menschlichem, bioptisch und autoptisch gewonnenem Lebergewebe bestätigen die Beobachtung von $\mathrm{B}$ e r g, daß der Inhalt nucleolärer Blasen mit Hilfe eines „Schleusenmechanismus“ aus dem Kern in das Protoplasma übertreten kann, ohne daß der Binnenraum des Kernes eröffnet wird. Auch ganze Nucleolen und umfangreiche nucleoläre Blasen werden gelegentlich auf gleiche Weise in den Zelleib abgegeben. Solche Vorgänge werden nur bei abnormer Vergrößerung des Kernes' beobachtet und sind deshalb bei chronischer Hepatitis epidemica besonders häufig. Dies erklärt sich damit, daß stark hypertrophierte Kerne eine im Vergleich zu ihrem Volumen zu kleine Oberfläche haben, so daß eine ausreichende Stoffabgabe auf dem Wege der Diffusion nicht mehr möglich ist. Daher tritt kompensatorisch der Schleusenmechanismus in Tätigkeit. Gelegentlich ist der Inhalt der nucleolären Blasen basophil. In solchem Falle führt seine Abgabe ans Protoplasma zur Bildung basophiler Schollen, die ihre Färbbarkeit nach den Ergebnissen von B r a c het, O p i e u. a. dem Gehalt an Ribosenucleinsäuren verdanken. Darin wird ein neuer Beleg für die Vorstellung Casperssons über die Kernabhängigkeit cytoplasmatischer Nucleinsäuren erblickt. Die Bildung solcher ungewöhnlich großer basophiler Schollen in hypertrophierten menschlichen Leberzellen wird mit der Vermehrung zelleigenen Eiweißes in Verbindung gebracht, die normale diffuse Basophilie wird dagegen als Ausdruck der hepatozellulären Entstehung von Bluteiweißkörpern aufgefaßt.
\end{abstract}

$\mathrm{V}_{\mathrm{d}}$ or einer Reihe von Jahren hat Ber $\mathrm{g}^{1-3}$ an den Leberzellen der südamerikanischen Blindwühle Siphonops annulatus und des Menschen erstmalig einen Übertritt nucleolärer Substanzen aus dem Kern in das Protoplasma beschrieben,

* Vortrag, gehalten vor der Freiburger Medizinischen Gesellschaft am 19. Oktober 1948.

1 W. B e r g, Z. mikroskop.-anat. Forschg. 28, 565 [1932]. der auf sehr eigenartige Weise vor sich geht und morphologisch gut $\mathrm{zu}$ erfassen ist. Zunächst schwillt der von kleinen Chromatinbröckeln fast lückenlos besetzte Nucleolus zu einer anfangs dickwandigen, später dünnwandigen, schwach ge-

2 W. B e r g, Z. mikroskop.-anat. Forschg. 35, 146 [1934].

${ }_{3}$ W. B e r g, Z. mikroskop.-anat. Forschg. 38, 644 [1935]. 